\title{
3D Laser Structure Modification of Photochromic Nanocomposites Based on Porous Glass
}

\author{
Vadim VEIKO, Maksim SERGEEV and Galina KOSTYUK \\ ITMO University, 197101, 49, Kronverkskiy av., Saint Petersburg, Russia \\ E-mail: siriusmaks@gmail.com
}

\begin{abstract}
3D laser densification of photochromic porous glasses by IR radiation of fiber laser $(\lambda=\sim 1.07$ $\mu \mathrm{m})$ is realized. Modified areas of different shapes and sizes that formed in the bulk of photochromic porous glass (PCPG) plates are investigated by optical methods.
\end{abstract}

DOI: 10.2961/jlmn.2015.01.0004

Keywords: photochromic porous glass, glassy composite, laser-induced densification

\section{Introduction}

Laser-induced modification of silicate sol-gel materials [1, $2]$ and porous glasses (PG) $[3,4]$ is taking more and more interest last years.

Direct surface densification of PG by $\mathrm{CO}_{2}$-laser action was used for microlens arrays [4] and planar waveguides [5] fabrication. Unfortunately this wavelength doesn't allow realizing of 3D modification because of strong absorption by PG at 10,6 mcm wavelength.

Sugioka K. et al. proposed the 3D writing techniques for fabrication of microfluidic channels and cavities in the bulk of PG by femtosecond laser ablation $[6,7]$. In this case an ultrashort laser action leads to the destruction of PG structure. The direct formation of hollow microchannels and square-shaped microfluidic chamber embedded in $\mathrm{PG}$ plate immersed in water by femtosecond laser ablation was demonstrated in $[8,9]$. The formation of hollow microstructures in $\mathrm{PG}$ immersed in Rhodamine $6 \mathrm{G}$ dissolved in water at a concentration of $C=6.0 \times 10^{-3} \mathrm{~mol} / \mathrm{L}$ by nanosecond laser ablation is reported in [10]. Such impregnation of PGs leads to increasing of its optical absorption of laser radiation. The authors note that the PG opens broad possibilities for multifunctional integration by filling of pores by various materials [8]. This might be important for creation of novel devices based on laser micro- and nanofabrication.

The PGs immersed in nanoparticles of photosensitive elements $(\mathrm{Ag}, \mathrm{Cu}, \mathrm{Au}$, etc.) dissolved in a water at a certain concentration is of great interest to the scientific community due to their various potential applications in photonics [11-13]. These glass composite materials with MAs which were created in the bulk of material have unique linear and nonlinear optical properties. The effect of a plasmon resonance and a high optical response, as well as ferroelectric, electro-optical and magneto-optical properties are ones of the most important.

In this research we've used the fact that PG can be effectively impregnated by different impurities to change their optical properties $[14,15]$. Such process was applied by authors to increase an optical absorption for fiber laser radiation $(\sim \lambda=1.07) \mu \mathrm{m})$ in the bulk of PG $[16,17]$. Finally we've considered the possibility of $3 \mathrm{D}$ near infrared fiber laser-induced modification of photochromic PG glass impregnated with silver and copper halides.

\section{Experiments}

Fabrication of PCPG consisted of two main chemical stages: a through-leaching of two-phase alkaliborosilicate glasses and an impregnation of the received PG according to the technique described in $[18,19]$. The PCPG plates with composition (by analysis, wt \%): $0.61 \mathrm{Na}_{2} \mathrm{O}-$ $3.36 \mathrm{~B}_{2} \mathrm{O}_{3}-94.30 \mathrm{SiO}_{2}-1.22 \mathrm{Ag}_{2} \mathrm{O}-0.04 \mathrm{CuO}-0.47$ $\mathrm{K}_{2} \mathrm{O}$ [19] were received in the result.

The next processing stage of PCPG is a laser irradiation. The modification of optical properties takes place in the bulk of PCPG plates at chemical processing. The formation of modified areas (MAs) in the bulk of PCPG was realized by $\mathrm{CW}$ (power up to $15 \mathrm{~W}$ ), as well as by pulsed radiation (average power up to $10 \mathrm{~W}$ ) of ytterbium fiber laser at $1.07 \mu \mathrm{m}$ wavelength. The pulsed fiber laser has a pulse duration $t_{p}=100 \mathrm{~ns}$ and pulse repetition rate $f=50$ $\mathrm{kHz}$.

The spherical MAs (with stationary laser beam), and cylindrical MAs (with scanning laser beam) were formed depending on the conditions of laser irradiation. The size and shapes of the MAs were determined by laser power, duration of action, and size of beam waist, which was arranged in the bulk of PCPG plate at a depth of 150-200 $\mu \mathrm{m}$ from the surface. A $10 \times$ objective with a numerical aperture of 0.25 (the focusing length is $4.75 \pm 0.25 \mathrm{~mm}$ ) was used for focusing the beam into the PCPG plate. Formation of MAs with cylindrical shape was made at a motion of PCPG plate with $18 \mu \mathrm{m} / \mathrm{s}$ speed.

The optical power of the incident $\left(P_{0}\right)$ and transmitted $\left(P_{\tau}\right)$ laser radiation was recorded with the use of a Gentec Solo-2M optical power measuring instrument with a UP19K-110F-H9 pyroelectric power detector. Measurement of $P_{0}$ and $P_{\tau}$ allowed estimating of absorption ability of PCPG during the formation of MA. The temperature in the zone of a laser action on a surface of sample was recorded with the use of a Flir Titanium $520 \mathrm{M}$ thermal vision camera. After laser processing the PCPG plates with MAs were investigated with use of optical microscope Axio Imager Carl Zeiss in transmitted light with a magnification of $100 \times$. The optical densities of PCPG and MAs in the wavelength range of $350-900 \mathrm{~nm}$ were obtained by microspectrophotometer MSFU-K U-30.54.072. 
Highly developed structure of the PCPG determines its unique adsorption properties. This fact causes a change of its optical properties with time in the case of air storage $[3,5]$. After the laser irradiation, the PCPG plates with MAs were sintered in electric furnace in air to close the pores and to stabilize its properties. The sample of PCPG was annealed at $870{ }^{\circ} \mathrm{C}$ for 4 hours to remove the pores. Final optical characteristics of photochromic quartz-like glass (PCQG) plates with MAs were formed by thermal processing in a furnace [5].

\subsection{Laser-induced bulk modification results}

Laser-induced recording of spherical MAs was carried out in the bulk of the fixed PCPG plate at the power of incident $\mathrm{CW}$ radiation $\left(P_{0}\right): 4.4,6,8,11.5$ and $13 \mathrm{~W}$ and corresponding exposure time $\left(t_{e x}\right): 5,10,15$ and $20 \mathrm{~s}$. The result of the experiments was determined by the irradiation regimes, leading to formation of MAs with clear boundaries and sizes in the range from 44 to $135 \mu \mathrm{m}$. These areas were able to focus a transmitted radiation $\left(P_{0}=4.4 \mathrm{~W}\right.$ at $t_{e x}=15 \sim 20 \mathrm{~s}, \quad P_{0}=6 \sim 11.5 \mathrm{~W}$ at $t_{e x}=5 \sim 20 \mathrm{~s}$ and $P_{0}=13 \mathrm{~W}$ at $t_{e x}=5 \mathrm{~s}$ ). Further increase of the laser power and exposure time resulted in formation of MAs with clear boundaries and sizes in the range from 178 to $311 \mu \mathrm{m}$ $\left(P_{0}=13 \mathrm{~W}\right.$ at $\left.t_{e x}=10 \sim 20 \mathrm{~s}\right)$. Irreversible structural changes in the beam waist were observed with further increase of the radiation power and exposure time. These changes leaded to a deterioration of optical characteristics, in particular to degradation of PCPG transparency and destruction at further irradiation. Spherical MA formed in the bulk of PCPG at laser power $P_{0}=13 \mathrm{~W}$ at $t_{e x}=15 \mathrm{~s}$ are shown in fig. 1.

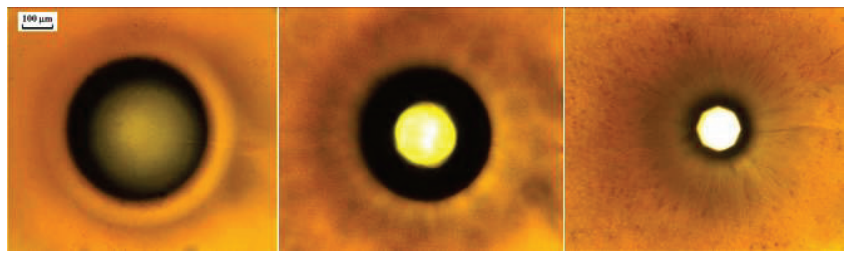

Fig. 1. Optical images of the spherical MA (after the sintering in furnace) formed in the bulk of PCPG. a, b, c - different positions of the focal plane of the microscope in transmitted light: a - surface of PCPG plate; $b$ - the center of MA; $c$ - image of the condenser aperture of microscope illumination system.

The optical images of spherical MA in the bulk of PCPG plate were obtained at different positions of the focal plane of the microscope in depth of the glass plate in transmitted light. The MAs are situated under the surface (fig. 1.a). The region with brighter color which surrounded by a dark ring was observed, when the fine focal plane of the microscope intersected with center of MA (fig. 1.b). Thus, the light of the microscope illumination system passed through the central part of MA and strongly dissipates at its edges. At this, the boundary layer of MA acted as a diaphragm, limiting the light transmitted through MA. The image of the condenser aperture of microscope illumination system was obtained by means of MA (fig. 1.c). Thus, MA together with microscope lens operated as an optical system with a modified focal length. This fact can give evidence of the different values of refractive index of MA and PCPG plate. The size of MA decreased from 270 to $200 \mu \mathrm{m}$ after the sintering and from 595 to $465 \mu \mathrm{m}$ considering the boundary layer of the annular structure of MA. The optical properties of MA in the bulk of PCPG plate after sintering in furnace were saved.

The cylindrical MAs were recorded at the power of incident $\mathrm{CW}$ radiation: 4.4, 5.2, 6.1 and $7.9 \mathrm{~W}$ for single scan. Cross-sectional size of cylindrical MAs ranged from 38 to $68 \mu \mathrm{m}$ in dependence to an incident radiation power. Formations of MAs were not observed at a smaller power of laser radiation in a single scan. Reduction of crosssectional size of cylindrical MAs was achieved by decrease of the incident $\mathrm{CW}$ radiation power down to $3.5 \mathrm{~W}$ and increase of the number of scans up to 5 (fig.2.a). It is possible to receive MAs with the size of $8 \mu \mathrm{m}$. Further reduction of MAs size was obtained with decrease of an incident radiation power and with increase of number of passes by deceleration of the sample movement and by applying a pulsed radiation for the laser processing. Reducing of colloidal silver particles concentration in the PG channels contributed to a reduction of cross-sectional size of cylindrical MAs as well [16].

The MAs of cylindrical shape with cross-sectional dimensions of 5-12 $\mu \mathrm{m}$ were formed with the use of pulsed radiation in the bulk of PCPG with lower concentration of silver particles (fig. 2. b, c). Thus, the writing of MA with transverse size of $11 \mu \mathrm{m}$ was carried out at average power of pulsed radiation $2 \mathrm{~W}$ and 3 scans (fig. 2. b). The MAs with Y-shape and transverse size of 5-9 $\mu \mathrm{m}$ was formed at average power of pulsed radiation $3.25 \mathrm{~W}$ at single scan (fig. 2. c).

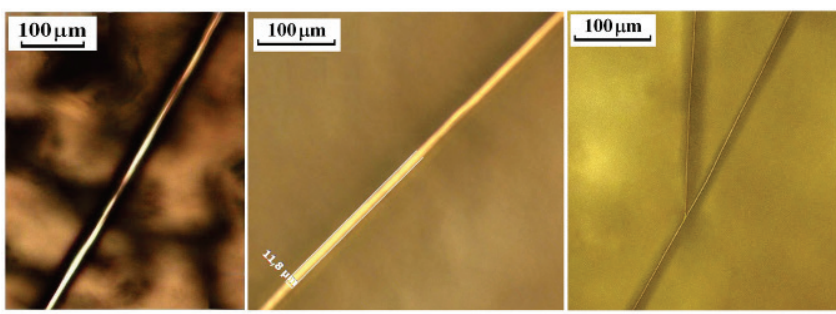

Fig. 2. Optical images of the cylindrical MAs $(a, b)$ Y-shaped MA (c) was formed in the bulk of PCPG for several passes with scanning rate of $18 \mu \mathrm{m} / \mathrm{s}$.

The surface temperature in the center of the processing area was registered by thermal vision camera. Measured values of temperature were $\sim 750-850{ }^{\circ} \mathrm{C}$, when the irradiation process at MAs formation was stationary. Such relatively high value of temperature is provided by corresponding laser exposure parameters and by relatively high absorption of the PCPG $(A)$. It can be evaluated by the expression [20]:

$$
A \approx(1-2 R)\left[1-\frac{P_{\tau}}{P_{0}(1-2 R)^{2}}\right],
$$

where $\mathrm{R}$ is $\mathrm{PCPG}$ plate reflectance. At $\mathrm{R} \sim 0.04, A$ is equal to $\sim 0.37$.

In the experiments the power density was changed in the range of $0.97-2.85 \mathrm{MW} / \mathrm{cm}^{2}$ for the $\mathrm{CW}$ laser radiation and the average power density was changed in the range 18.0$54.0 \mathrm{~kW} / \mathrm{cm}^{2}$ for the pulsed radiation. The temperature in the bulk of PCPG in the focal spot can be estimated as [14]: 


$$
T \approx \frac{A q r_{0}^{2}}{4 k \delta} \ln \left(19.4 \frac{a t}{r_{0}^{2}}\right)+T_{0},
$$

where $t$ is a processing duration (from 5 to $25 \mathrm{~s}$ ); $k$ is a thermal conductivity of PCPG $(\sim 3.3 \mathrm{~W} /(\mathrm{m} \cdot \mathrm{K})) ; \delta$ is a depth of light penetration $\left(\sim 1.5 \times 10^{-3} \mathrm{~m}^{-1}\right) ; a$ is a thermal conductivity $\left(\sim 10^{-5} \mathrm{~m}^{2} / \mathrm{s}\right)$. Calculated value of temperature is up to $\sim 850-1000{ }^{\circ} \mathrm{C}$.

The absorption of incident radiation by silver halide particles and energy transfer to the glass network is the basis of PCPG heating mechanism. It is known that the crystallization, change of optical properties and destruction of multicomponent glasses is affected by laser exposure [21]. These modifications are based on similar heating mechanism. In our case, the modification of PCPG was implemented through process of porous structure termodensification at the beam waist without structural-phase transitions and destruction of material.

Thus, the modification of optical properties of glass without the destruction and the structural-phase transitions in the exposed region becomes possible at definite temperature range and heating / cooling rates of glass. The regime of heating is determined both by parameters of laser processing and the concentration of metal particles in the glass (and also by its distribution in the volume). The absorption capacity of glass as well as its crystallization temperature depends on the concentration of metal particles and its distribution in the glass, too.

\subsection{Alteration of material properties after processing}

Optical density $(D)$ of PCPG plates and MA was estimated by measurement of spectral transmittance $(\tau)$ in the photon energy range from 1.38 to $3.55 \mathrm{eV}$ (or wavelength range from 350 to $900 \mathrm{~nm}$ ):

$$
D=-\lg \tau \text {. }
$$

In addition, the relative optical density $\Delta D$ (fig. 3) was determined. This value is equal to the difference of optical density $(D)$ of the objects under study (PCPG or MA) and the optical density of fused quartz $\left(D_{Q}\right)$ that was chosen as the standard at the process of recording:

$$
\Delta D=D-D_{Q} .
$$

The additional absorption in photochromic glass can be initiated under the action of UV or NIR radiation [22]. In irradiated regions the precipitation of colloidal silver particles, which are the color centers with a typical size of $60 \mathrm{~nm}$, occurs. The absorption band of these particles with spherical shape corresponds to the range of $E_{p}=2.48-2.59$ $\mathrm{eV}$. The formation of oblate ellipsoidal particles, which give the absorption in the long-wave range of spectrum with lower photon energy, is possible together with formation of spherical particles [23]. The UV laser irradiation of the PCQG plates leads to the appearance of plasmon resonance peaks (in the range of $E_{p} \sim 3.10-3.55 \mathrm{eV}$ ). These peaks are characteristic for quasi metallic silver particles $[11,22,23]$. Spectral dependences of the optical density of investigated PCPG plates in irradiated (i.e. MA) and non irradiated regions prove the presence of colloidal silver particles (fig. 3, curve 1 and 2).

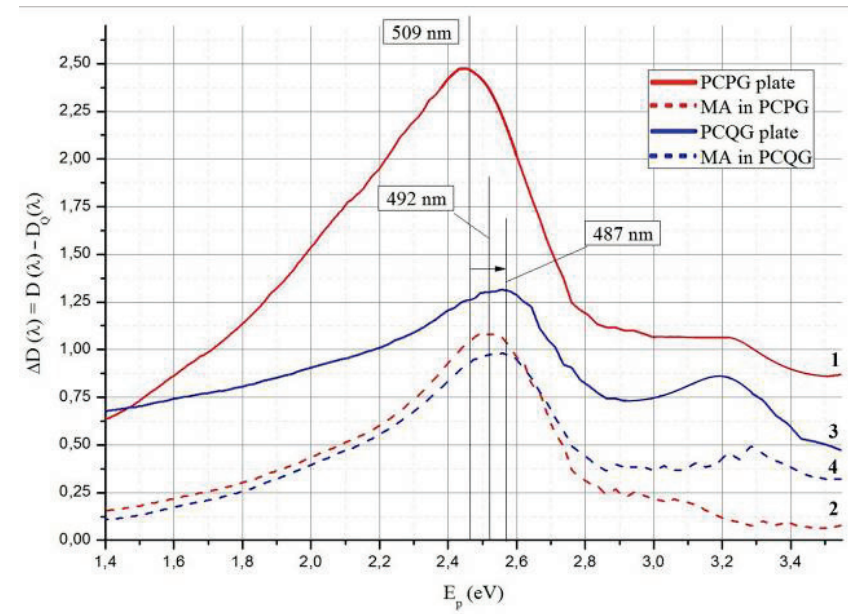

Fig. 3. Spectral dependences of the optical density of (1) the PCPG plate, (2) the MA, and the PCQG plate obtained after sintering of (3) the PCPG and (4) the MA formed in the PCPG plate (compared with the optical density of the fused quartz).

It can be seen from the comparison of the spectral dependence of $\triangle D$ of the PCPG plate and the MA in the full examined range $\left(E_{p}=1.38-3.55 \mathrm{eV}\right)$ that $\triangle D$ of MA is substantially lower than $\triangle D$ of the PCPG plate (fig. 3, curve 1 and 2). Probably, the cause of these changes is smaller number of diffusers which are silver particles. The decrease of the particles size to values less than wavelength of the visible light after laser irradiation can be the cause too. In addition, the spectral dependence of optical density MA demonstrates a small (about 0.08-0.1 eV) displacement of its peak in the direction to UV region. This fact may be associated to the shape and orientation of the silver particles [11].

The optical characteristics were investigated again after the sintering of PCPG plates with MAs formed in the bulk in furnace for a purpose of PCQG formation. A comparison between optical densities of PCQG (fig. 3, curve 3) and PCPG plates (fig. 3, curve 1) suggests that, after sintering, the general character of the dependence did not change. Nevertheless, the maximum value of the optical density was reduced by a factor of 1.8 (from 2.4 to 1.3 ) and a peak position was shifted to the near-UV range. A character of optical density dependence for MA after sintering of the PCPG with the MA barely changes (fig. 3, curve 4 compared to curve 2). Dependences of the optical density of both PCQG plate and MA are shown in fig. 3, curves 1 and 2. The shift of peaks to the same photon energy (wavelength) is observed $\left(E_{p}=2.55 \mathrm{eV}\right)$. This fact can be attributed to the reduction of silver particles quantity in the irradiated region (i.e. MA) and in the entire PCPG plate after sintering in a furnace.

\section{Conclusions}

3D laser modification of properties of PCPG plates impregnated with silver halides is presented. The $\mathrm{CO}_{2}$ laser light is not suitable for modification in the bulk of PG, because it is not able to penetrate into the volume due to strong absorption. Impregnation of PG plates with metal particles (1.22-2.4\% Ag) allowed an increase of absorptivity of photochromic porous glass (PCPG) up to 0.224 at a wavelength $(1.07 \mu \mathrm{m})$ for which initial PG is transparent. It was enough for near IR light absorption enhancement, suf- 
ficient for modification in the bulk of PCPG which was implemented by the example of volume thermal densification under laser irradiation. Process of modification PCPG properties takes place in the waist of laser beam and leads to local densification of the glass network of PG. Laserinduced thermal densification occurs due to a heating of PCPG by metal particles which absorbs radiation within a glass network.

The MAs were formed in the bulk of PCPG plates with various shapes and sizes by NIR radiation of fiber laser with a use both CW and pulsed irradiation. Regions with spherical shape with diameters from 50 to $350 \mu \mathrm{m}$ and regions with cylindrical shape with cross-sectional diameter from 5 to $70 \mu \mathrm{m}$ were produced. Optical properties (transmittance and optical density) of PCPG and MAs before and after sintering of glass plate in furnace were investigated.

\section{Acknowledgments}

The study was supported by Grant for leading universities of RF (subsidy 074-U01) and Russian Federation President Grant for leading scientific school № NH 1364.2014.2

Authors are grateful to T. V. Antropova, I. N. Anfimova and M. A. Girsova for manufacturing of PCPG plates.

\section{References}

[1] J. D. Musgraves, K. Richardson and H. Jain: J. Opt. Mat. Exp., 1(5), (2011) 921-935.

[2] L. C. Klein: "Sol-gel optics: processing and applications" ed. (Springer, New York, 1994) p. 583.

[3] D. Enke, F. Janowski, W. Schwieger: Microporous Mesoporous Mater., 60, (2003) 19-30.

[4] V. P. Veiko, E. B. Yakovlev, V. A. Chuiko: Chemical Processing of Advanced Materials. Edited by L.L.Hench and Jon K. West, John K. Wiley and Sons Inc.,(1992) p.919-931.

[5] T. V. Antropova, V. P. Veiko, G. K. Kostyuk, M. A. Girsova, I. N. Anfimova, V. A. Chuiko and E. B. Yakovlev: J. Glass Phys. Chem., 38(6), (2012) 478-490.

[6] K. Sugioka, Y. Cheng: J. Lab Chip., 12, (2012) 35763589.

[7] K. Sugioka, Y. Cheng: "Femtosecond Laser 3D Micromachining for Microfluidic and Optofluidic Appli- cations", (Springer Briefs in Applied Sciences and Technology, London, 2014) p.129.

[8] Y. Liao, Y. Ju, L. Zhang, F. He, Q. Zhang, Y. Shen, D. Chen, Y. Cheng, Z. Xu, K. Sugioka, K. Midorikawa: J. Opt. Lett., 35, (2010) 3225-3227.

[9] Y. Ju, Y. Liao, L. Zhang, Y. Sheng, Q. Zhang, D. Chen, Y. Cheng, Z. Xu, K. Sugioka, K. Midorikawa: J. Microfluid Nanofluid, 11, (2011) 111-117.

[10] C. Liu, Y. Liao, F. He, Y. Shen, D. Chen, Y. Cheng, Z. Xu, K. Sugioka, K. Midorikawa: J. Opt. Express, 20(4), (2012) 4291-4296.

[11] M. Sendova and J. A. Jimenez: J. Phys. Chem. C., 116, (2012) 17764-17772.

[12]A. Stalmashonak, G. Seifert, A. Abdolvand: "Ultrashort pulsed laser engineered metal-glass nanocomposites", (Springer Briefs in Physics, New York, 2013) p. 70 .

[13]F. Goutaland, M. Sow, N. Ollier, F. Vocanson: J. Optical Materials Express, 2(4), (2012) 350-357.

[14] G. K. Kostyuk, M. M. Sergeyev, E. B. Yakovlev: J. Glass Phys. Chem., 39(5), (2013) 480-489.

[15]R. A. Zakoldaev, M. M. Sergeev, G. K. Kostyuk: J. Proc. SPIE., 9065, (2013) 90650P.

[16] M. M. Sergeev, G. K. Kostyuk: J. Proc. SPIE. 9065 , (2013) 90650Q.

[17] M. M. Sergeev, G. K. Kostyuk: J. Glass and Ceramics, 4, (2014) 07-13.

[18] M. A. Girsova, I. A. Drozdova and T. V. Antropova: J. Glass Phys. Chem., 40(2), (2014) 162-166.

[19] M. A. Girsova, T. V. Antropova, I. A. Drozdova, L. N. Kurilenko, I. G. Polyakova and A. S. Pasishnik: Third Int. Sc. Conference "Nanostructured Materials 2012: Russia-Ukraine-Belarus 'NANO-2012'”, SentPetersburg, (2012) p.224.

[20] I. Miyamoto, K. Cvecek, Y. Okamoto, M. Schmidt, H. Helvajian: Opt. Expr., 19(23), (2011) 22961-22973.

[21] T. Honma, Y. Benino, T. Fujiwara and T. Komatsu: J. Appl. Phys. Lett., 88, (2006) 231105.

[22] L. B. Glebov, N. V. Nikonorov, G. T. Petrovsky: Autometriya, 5, (1988) 33-46.

[23] G. K. Kostyuk, M. M. Sergeev, M. A. Girsova, E. B. Yakovlev, I. N. Anfimova and T. V. Antropova: J. Glass Phys. Cem., 40(4), (2014) 415-420.

(Received:August 28, 2014, Accepted: November 28, 2014) 